\title{
Image-based Optical Miniaturized Three-Axis Force Sensor for Cardiac Catheterization
}

\author{
Yohan Noh, Hongbin Liu, Sina Sareh, Damith Suresh Chathuranga, Helge Wurdemann, Kawal Rhode, \\ Kaspar Althoefer
}

\begin{abstract}
In order to determine the cause of and to treat an abnormal heart rhythm, electrophysiological studies and ablation procedures of the heart sensorized catheters are required. During catheterization, force sensors at the tip of the catheter are essential to provide quantitative information on the interacting force between the catheter tip and the heart tissue. In this study, we are proposing a small sized, robust, and lowcost three-axis force sensor for the catheter tip. The miniaturized force sensor uses fiber-optic technology (small sized multi-cores optical fiber and a CCD camera based on image processing to read out the forces by measuring light intensity which are modulated as a function of the applied force. In addition, image processing techniques and a Kalman filter are used to reduce the noise of the light intensity signals. In this paper, we explain the design and fabrication of our three-axis force sensor and our approach for reducing noise levels by applying a Kalman filter model, and finally discuss the calibration procedure. Moreover, we provide an assessment of the performance of the proposed sensor.
\end{abstract}

\section{INTRODUCTION}

Electrophysiological studies which investigate the cause of abnormal heart rhythm (cardiac arrhythmias) in cardiovascular patients usually involve catheterization. The test procedure is performed through passing a thin catheter into the right or left chambers of the heart, where the catheter tip can measure the location of electric pathways and ablate areas of the heart wall during an ablation process, in order to regulate faulty electric pathways [1]. Having completed electrophysiological measurements, ablation of identified points in the heart wall through electrodes at the catheter tip is carried out to remove the part of tissue which causes the abnormal heart rhythm using radiofrequency energy. Although catheter ablation has been carried out in patients for a long time, the success rate of ablation therapies has been very poor, around $15-45 \%$, and the patients often require further ablation procedures after the initial treatment [2 and $3]$. Several studies showed that the success rate of the ablation therapies strongly depends on a good mechanical contact between the electrode and the heart tissue [4]. The cardiac catheterization is usually performed under X-ray fluoroscopy

Manuscript received Oct $22^{\text {nd }}, 2015$. The research leading to these results has received funding from the European Commission's Seventh Framework Programme; project STIFF-FLOP (Grant No: 287728).

Yohan Noh, Hongbin Liu, Helge Wurdemann, and Kaspar Althoefer are with Centre of Robotics Research, King's College London, School of Natural and Mathematical Sciences, Department of Informatics, UK to enable real-time tracking of the catheter tip position. There are also works reported using magnetic resonance imaging (MRI) [5-8]. However, cardiologists are usually not able to reliably monitor the catheter tip force applied to the heart tissue [9]. Although some force is fed back to the cardiologist via the handheld catheter, the forces perceived are heavily distorted by the multitude of friction forces induced by blood vessels through which the catheter advances. Real-time images from X-ray and/or MRI are helpful in this context, but cannot provide detailed force information. The interaction forces between the electrode and the heart tissue is the essential information determining the success of the ablation procedure. Catheter tip force sensing not only improves the catheterization performance but also, prevent traumatization caused by the tip force on the heart or blood vessel (vein and artery) while advancing the catheter to the heart [10]. In-line with these requirements, we propose a force sensor to be integrated with the catheter tip to provide quantitative force feedback to the doctors during cardiac catheterization. A variety of miniaturized uni-axial and multi-axial catheter tip sensors based on piezoresistive materials [11], strain gauges [12], polyvinylidene fluoride (PVDF) films [13], and fiberoptics [14-16] have so far been proposed in the literature [6 and 7].

In this paper, we propose a new miniature three-axis force sensor which is integrated with the tip of a cardiac catheter to provide the tip contact force information. The proposed sensor consists of a $3 \mathrm{D}$ printed deformable structure and a 4core fiber bundle. The sensor can be assembled by plugging the fiber bundle into the sensing structure. The light intensity is modulated using a digital USB camera to obtain the three axial forces between the catheter tip and the cardiac tissue. Compared to existing three axial force sensors for catheter tip, the proposed design has the advantages of simpler structure, easier for fabrication and sensor calibration and lower cost. The fiber optic sensors [17-19] are free of electrical current. And, hence, the sensing is not influenced by the electromagnetic field (as it occurs during MRI-guided catheterization) or by the radio frequency power used for heart ablation. In addition, the sensor signal (light intensity) is insensitive to the environmental temperature (an issue when using Fiber Bragg Grating based methods) [20,21].

(e-mail *corresponding author: yohan.noh@kcl.ac.uk).

Sina Sareh is with the Department of Aeronautics, Imperial College London, UK.

Damith Suresh Chathuranga is with the Department of Robotics, School of Engineering, Ritsumeikan University, Shiga, Japan. 
Furthermore, the use of optic imaging immunes the sensor against the electromagnetic interference.

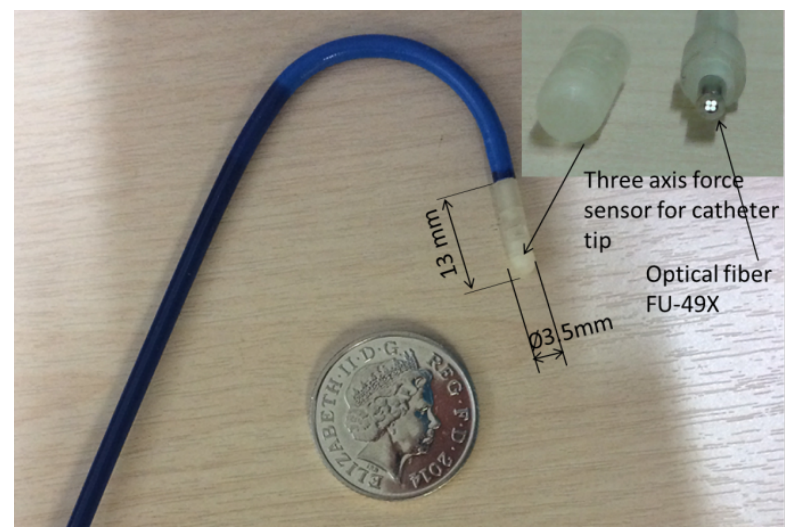

Fig. 1. The prototype of the three axis force sensor for catheter tip

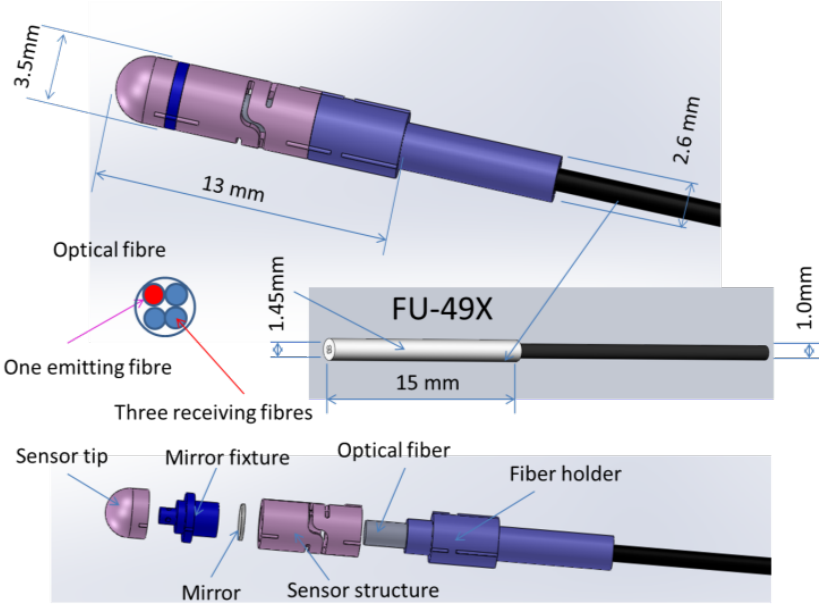

Fig. 2. Assembly overview of the three -axis force sensors for catheter's tip, integrating a FU-49X optical fiber.

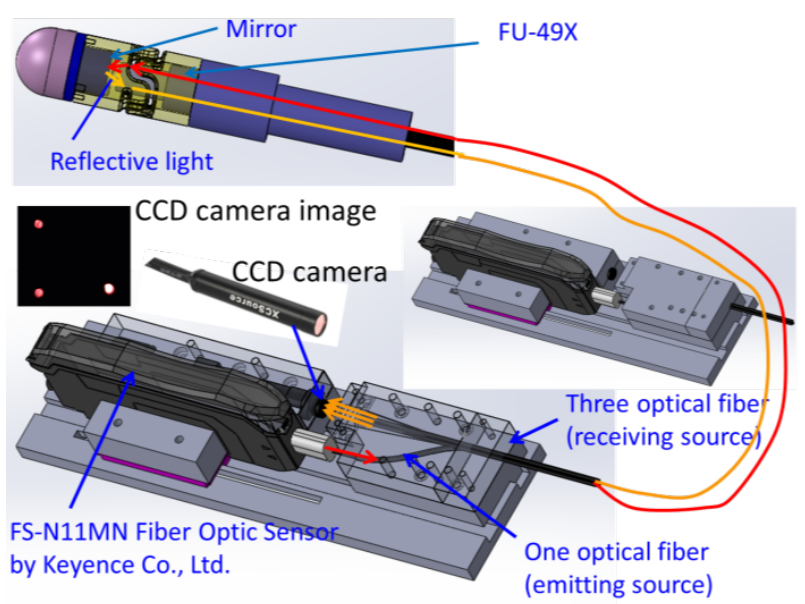

Fig. 3. The configuration of the sensor principle overview for three -axis force sensors for catheter's tip.

To date, a number of fiber optic force sensors have been developed. In [6], a three-axis catheter tip force sensor based on optical fibers and opto-couplers is proposed. This sensor use non-metallic components, thus is MRI compatible. Although this sensor achieved miniaturization of the overall size of the force sensor tip to $4 \mathrm{~mm}$ in diameter, the assembly

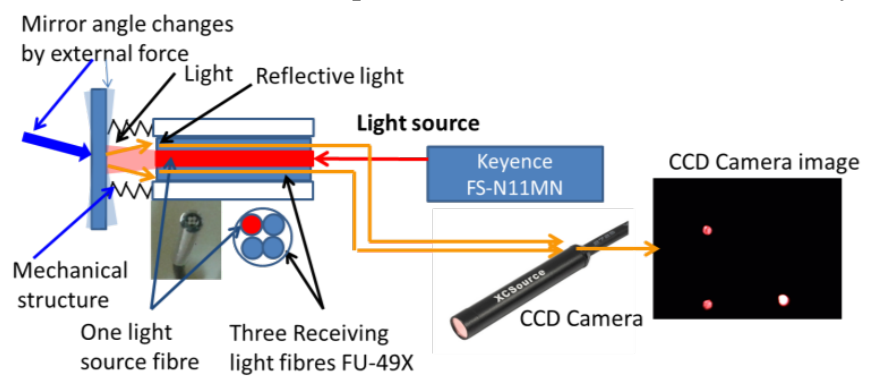

Fig. 4. Simplified principle overview of the three axis force sensors for catheter's tip.

and the integration of the tip sensor with the catheter is complex because individual three optical fibers need to be carefully inserted and accurately placed in front of the moving reflectors, increasing the manufacturing complexity. In [4], a three-axis force sensor based on optical fibers using the Fiber Bragg Grating (FBG) technique is proposed. Three-axis force sensing developed based on FBG has been reported in [21, 22]. The advantage of the FBG based sensor is that the sensor can be made into diameter less than $1 \mathrm{~mm}$, thus is applicable for integrating with biopsy needles [23]. However, this technology has much higher cost compared to our approach based on light intensity modulation due to the high cost of the interrogator. Also, the measured FBG sensor signals are highly influenced by temperature, and temperature compensation needs to be provided for, negatively impacting on design simplification [24].

Fiber optic technology and camera based force sensor have been proposed to measure multi-axis force/torque sensor. In $[25,26]$, a three axial force sensor is developed based on the measurement of the position of the centroid of light reflection in the image. However, this sensing method requires larger sensing structure and an optic fibre capable of transmitting images, thus is difficult to be miniaturized for catheter tip integration and has much higher cost. In addition, in [27-28], fiber optic technology and CCD camera based force sensors have been proposed to measure tactile information. However, this principle needs large deflection (deformation) to get large difference of pixel numbers. The large deflection of the sensing structure result in the difficulty to further miniaturize the sensor be integrated to the catheter tip.

In this paper, we propose a new design of three-axial force sensor which contains a $1.45 \mathrm{~mm}$ diameter 4 -core fiber bundle and simple sensing structure. The structure requires small deflection (deformation) to obtain sufficient sensitivity of force measurements (different method from camera image pixel numbers transferred from light intensity [27-28]). This approach simplifies the integration of the force sensor with the catheter tip, and is cheaper in comparison with any other approaches mentioned above. The miniaturized force sensing arrangement presented here can be easily integrated to many medical and industrial devices.

In this paper, we present the design and development of a three axis force sensor for the catheter tip based on the fiber 
optic technology using light intensity to transmit force signals. In the following, we explain how to design, fabricate and calibrate the sensor device, and subsequently calculate the

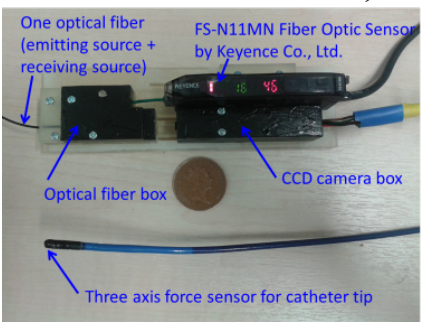

(a)

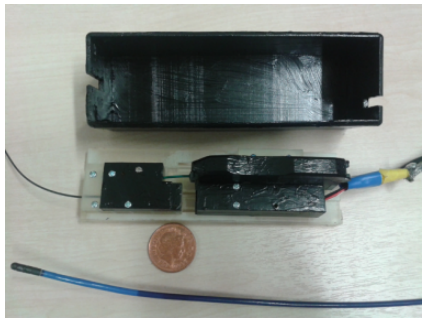

(c)

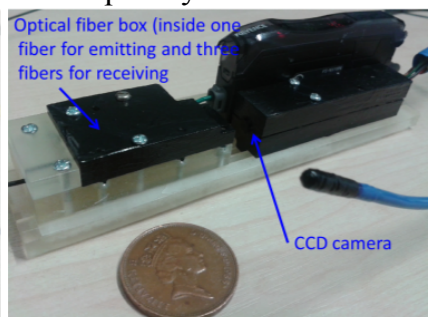

(b)

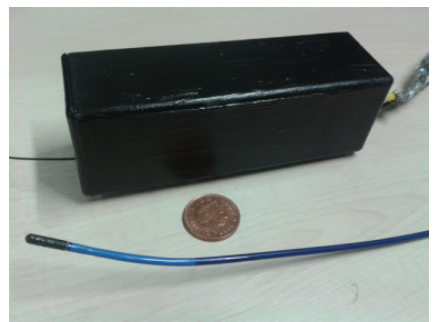

(d)
Fig. 5. Assembly overview of the camera box and optical fiber box: a) camera box and optical fiber box are tightly fixed with optical fiber and camera lens position when external force is applied on the camera cable and optical fiber; b) enlargement of the camera box and optical fiber box (camera faces to the three optical fibers for the receiving light); c) blocking external light using an external black cover; d) assembled cover with the optical fiber box and the camera box, and catheter tip' sensor

force components using a calibration process. The experimental results show that the proposed sensor has good performance in measuring three-axis force.

\section{Design Methods And SEnSor Structure}

\section{A. Design Concept}

The design concept of the multi-axis force sensor for the catheter tip should satisfy several conditions as follows:

1) The sensor should be scalable so that it can be integrated into the catheter tip. It is worth noting that this sensor can be useful for a variety of medical and industrial applications, e.g. a surgical robot tip.

2) The sensor should be capable of measuring three components of external force including $F_{\mathrm{x}}, F_{\mathrm{y}}$, and $F_{\mathrm{z}}$ in order to determine relevant interactions with the environment. The optimum contact force required to achieve a good and safe ablation line in RF procedures is around $0.2 \mathrm{~N}-0.3 \mathrm{~N}(20-30$ gm of force) [4, 6, and 7]. Sensor should be designed to withstand the forces expected in procedure. To protect the sensor from accidental overloads, the range of the sensor should be in the range of $F_{\mathrm{z}}= \pm 1.5 \mathrm{~N}, F_{\mathrm{x}}$ and $F_{\mathrm{y}}= \pm 0.5 \mathrm{~N}$. Additionally, the human heart normally beats 60-100 times per minute [6]. As a result, the typical bandwidth of operation for a force sensor should be at least $2 \mathrm{~Hz}$ for use in such a dynamic environment [6].

3) The sensor should be MR compatible, and free of electric currents and metal components.

\section{B. Configuration of Fiber Optic Multi-axis Force Sensor}

The structure of optical three-axis force sensor for the catheter tip is shown in Figs. 1 and 2. The sensor uses optical fiber (Keyence Corp., FU-49X, minimum fiber allowable bend radius $(\mathrm{R}=4 \mathrm{~mm}(0.157 "))$, a FS-N11MN Fiber Optic Sensor (Keyence Corp.), a mechanical sensor structure, a reflector, and a CCD camera as shown in Figs. 1 to 5. Figs. 1 and 2 show the FU-49X with its four small-sized optical fibers and a surrounding jacket. This arrangement uses a FS$\mathrm{N} 11 \mathrm{MN}$ as the light source to transmit constant light via one of the four fibers of the FU-49X to a mirror in the sensing structure (in general, we can use any light as the light source); the other three optical fibers are fixed to face the CCD camera as shown in Figs. 3 to 5; each of these three optical fibers send the light reflected by the corresponding mirror to the CCD camera. The CCD camera can acquire the light intensity of the three fibers - the light intensity varies as a function of deflections $\delta_{1}, \delta_{2}$ and $\delta_{3}$ of the three cantilever beams, shown in Fig. 6. When an external force is applied on the upper plate of the sensor structure, each of the cantilever beams is deflected. From the measured deflections, as finally acquired by the CCD camera, and knowledge of the spring constants of the beams or a calibration procedure, the three force components can be calculated. External light produces light intensity noise, and, hence, the cases for the CCD camera and the three optical fibers have been painted with black color, to shield light sensitive structures from ambient light as shown in Figs. 3 and 5.

\section{Sensor Structure Design and Simulation}

To measure the three force components, three cantilever beams are used as shown in Figs. 6 and 7. To satisfy the force range requirements of the three axis force sensor as mentioned above, we have performed an FEM analysis using the SolidWorks Simulation tool. Fig. 7 shows the result of the FEM simulation, revealing that maximum deflections are $\delta_{z}=$ $0.06[\mathrm{~mm}], \delta_{x}=0.2[\mathrm{~mm}]$ and $\delta_{y}=0.2[\mathrm{~mm}]$, when the maximum forces $F_{z}=2[\mathrm{~N}], F_{x}=1[\mathrm{~N}]$ and $F_{y}=1[\mathrm{~N}]$, respectively, are applied to the sensor structure, Fig. 7. We conclude that the structure can support the force within the required range. The material property values in this simulation were set at: tensile modulus of $1283 \mathrm{MPa}$, mass density of $1020 \mathrm{~kg} / \mathrm{m}^{3}$, tensile strength of $42500000 \mathrm{~N} / \mathrm{m}^{2}$; these assumptions were based on information provided by the manufacturer of the rapid prototyping machine (PROJET VisiJet $^{\circledR}$ EX200, 3D SYSTEM Co., Ltd).

\section{LIGHT INTENSITY MEASUREMENT FROM IMAGE}

In order to quantify the three deflections from the camera image, the light intensities of the reflective light in each channel should be converted from the camera images to time variant continuous values, using digital image processing techniques. The light intensity value on the image varies as a function of the amount of deflection of three cantilever beams, Fig. 8. In this section, we discuss our approach for converting the camera image data to light intensity analog values. Since, 
the analog data includes large levels of noise, a noise model was built based on a Linear Gaussian-model. Subsequently, a Kalman filter was implemented to reduce noise levels.

\section{A. Measurement of Light Intensity of Extracted Image from CCD Camera}

Fig. 8a shows an RGB image extracted and processed in OpenCV to produce a grayscale image. Each grayscale image contains a particular amount of the light intensity information. As shown in Figs. 3 and 4, the light reflected by the mirrors from the light source is transmitted using three optical fibers, and then the camera captures the received light from each image frame. Each of the image frames show the light intensity of the reflected light beams at a given time, and is divided into the three separated sections (three ROSs (Region of Section) as shown in Fig. 8), each corresponding to an individual cantilever beam. Following a change in amount of the three axis force components exerted on the mechanical sensor structure, the corresponding light intensity values change. The pixels on each of the ROS are converted to values between 0 and 255 and summed up, subsequently the result is divided by the number of pixels in the image section, to produce the corresponding light intensity values

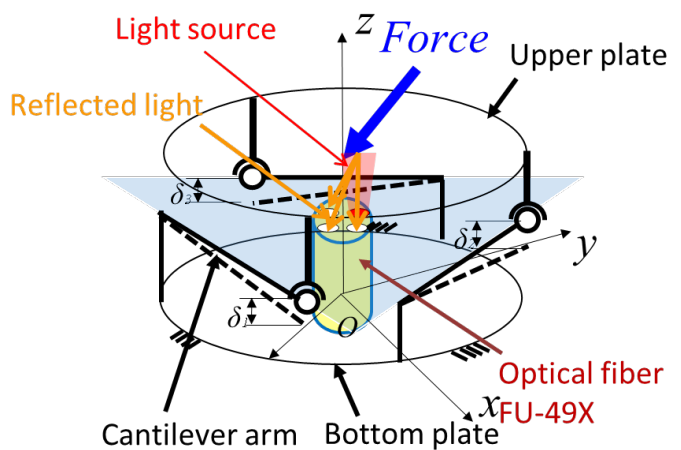

Fig. 6. The sensor mechanical structure based on the three cantilever beams

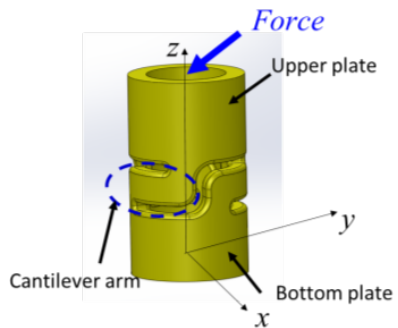

(a)

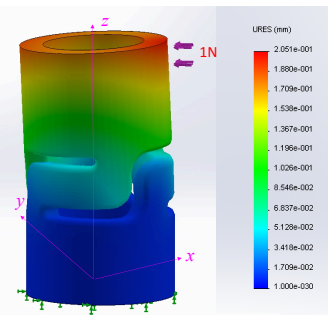

(c)

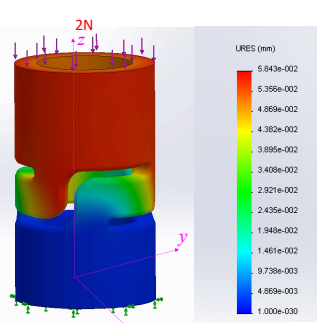

(b)

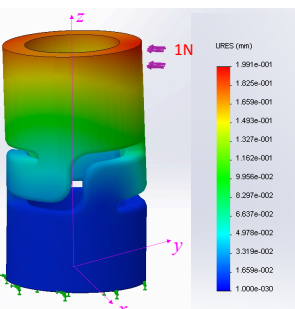

(d)
Fig. 7. FEA simulation of sensor mechanical structure using SolidWorks a) Sensor mechanical structure b) Maximum deflection when $F_{z}$ is applied along $\mathrm{z}$-axis c) Maximum deflection when $\mathrm{F}_{\mathrm{x}}$ is applied along $\mathrm{x}$-axis d) Maximum deflection when $\mathrm{F}_{\mathrm{y}}$ is applied along $\mathrm{y}$-axis

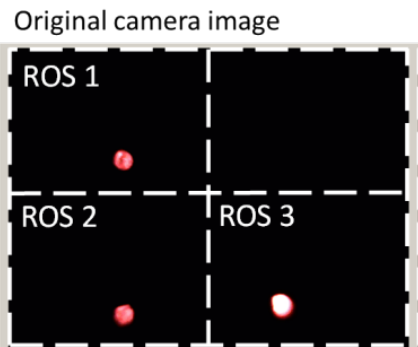

(a)
Processed image

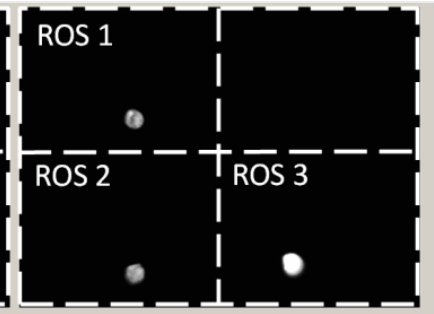

(b)
Fig. 8. Original and processed images, the captured by the camera, from three light channels: a) the original RGB image b) the grayscale image converted after applying median smoothing filter on the original RGB image.

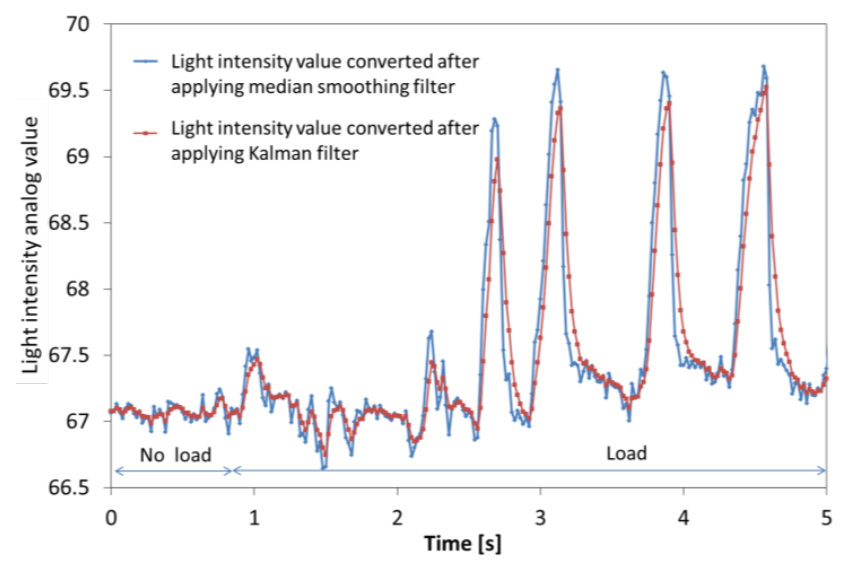

Fig. 9. Light intensity value converted after applying median smoothing filter and light intensity value filtered by Kalman filter

\section{B. Digital Image Processing to Reduce Noise}

The light intensity value of the grayscale image converted from the original RGB image includes large amount of noise. In order to reduce the noise levels, median smoothing filter is applied on the original RGB image, Fig. $8 \mathrm{~b}$ shows a grayscale image after applying the median smoothing filter on the original RGB image. The intensity value is obtained by averaging the pixel intensity of the selected ROS area.

\section{Linear Kalman Filter to Reduce Noise}

The noise produced by the image data acquisition from the CCD camera image is due to several reasons as the following: 1) the CCD camera, used in this study has low resolution (640 $\mathrm{x} 480,30 \mathrm{fps}$ ), 2) the optical fiber system involves light bend loss, and light intensity noise due to external light (despite using a camera box and an optical fiber isolation box to protect the optical fibers). To overcome this problem, we propose a Linear Kalman Filter to not only reduce the noise, but also to maintain the high signal updating rate.

The Linear Kalman filter is expressed in the following equation [29].

$$
\boldsymbol{x}_{t}=\boldsymbol{x}_{t-1}+\boldsymbol{w}_{t}
$$


where $\mathbf{x}_{\mathrm{t}}$ is the state vector of the light intensity, $\mathbf{w}_{\mathrm{t}}$ is the vector containing the process noise terms for each parameter in the state vector. The process noise is assumed to be white noise with the covariance matrix $\mathbf{Q}_{\mathrm{t}}$. Measurements of the system is expressed in Eq.2, according to the model [29]

$$
\boldsymbol{z}_{t}=\boldsymbol{H}_{t} \boldsymbol{x}_{t}+\boldsymbol{v}_{t}
$$

where, the measurement vector $\mathbf{z}_{\mathrm{t}}$ is the light intensity (after applying median smoothing filter), $\mathbf{H}_{\mathrm{t}}$ is the transformation matrix that maps the light intensity into physical quantity, $\mathbf{v}_{\mathbf{t}}$ is the vector containing the measurement noise terms for each observation in the measurement vector.

Like the process noise, the measurement noise is assumed to be Gaussian white noise with covariance matrix $\mathbf{R}_{\mathbf{t}}$. Here, the transformation matrix $\mathbf{H}_{\mathrm{t}}$ is set to 1 because the measurement vector $\mathbf{z}_{\mathrm{t}}$ and the state vector $\mathbf{x}_{\mathrm{t}}$ are same dimension, light intensity. The Kalman filter algorithm involves two stages: prediction and measurement update. The standard Kalman filter equations for the prediction stage are as follows:

$$
\begin{array}{r}
\widehat{\boldsymbol{x}}_{t}^{-}=\widehat{\boldsymbol{x}}_{t-1} \\
\boldsymbol{P}_{\boldsymbol{t}}^{-}=\boldsymbol{P}_{t-1}+\boldsymbol{Q}_{t}
\end{array}
$$

In the prediction stage, predict (priori) state estimate $\widehat{\boldsymbol{x}}_{t}^{-}$, and, predicted (a priori) estimate covariance matrix $\boldsymbol{P}_{\boldsymbol{t}}^{-}$can be obtained using Eqs. (3) and (6) with initial values such as $\widehat{\boldsymbol{x}}_{t-1}$ and $\boldsymbol{P}_{t-1}$ (in general we choose them to find better performance of Kalman filter) In the update stage, optimal Kalman gain $\boldsymbol{K}_{t}$, updated (a posteriori) state estimate $\widehat{\boldsymbol{x}}_{t}$ (true light intensity value predicted by Kalman filter), and update (a posteriori) estimate covariance matrix $\boldsymbol{P}_{t}$ can be updated using $\widehat{\boldsymbol{x}}_{t}^{-}, \boldsymbol{P}_{\boldsymbol{t}}^{-}$and measurement vector $\boldsymbol{z}_{t}$. $\hat{\boldsymbol{x}}_{t}$ and $\boldsymbol{P}_{t}$ are substituted to $\widehat{\boldsymbol{x}}_{t-1}$ and $\boldsymbol{P}_{t-1}$. These two stages are recursively predicting and updating using Eqs. 3 and 4 . The measurement update equations (5) to (7) are given by:

$$
\begin{array}{r}
\widehat{\boldsymbol{x}}_{t}=\widehat{\boldsymbol{x}}_{t}^{-}+\boldsymbol{K}_{t}\left(\boldsymbol{z}_{t}-\widehat{\boldsymbol{x}}_{t}^{-}\right) \\
\boldsymbol{P}_{t}=\boldsymbol{P}_{\boldsymbol{t}}^{-}-\boldsymbol{K}_{t} \boldsymbol{P}_{\boldsymbol{t}}^{-} \\
\boldsymbol{K}_{t}=\boldsymbol{P}_{t}\left(\boldsymbol{P}_{t}+\boldsymbol{R}_{t}\right)^{-1}
\end{array}
$$

Fig. 9 shows the light intensity value after applying Kalman filter from the original light intensity value applying median smoothing filter makes noise lower. In this study, the Kalman filter is tuned to achieve $2 \mathrm{~Hz}$ frequency response of the low noise force estimation with the process noise covariance matrix $\mathrm{Qt}=0.5$, and the measurement noise covariance matrix $\mathrm{Rt}=2.0$. The frequency response can be further increased, but with the trade off increasing the noise level.

\section{SENSOR CALIBRATION}

\section{A. Sensor Calibration}

In the previous section, we converted image pixel value into the light intensity value. However, the light intensity value need to be converted further to physical force information. Therefore, sensor calibration is used to convert acquired measurement signals into physical quantities. The calibration process aims at obtaining a relationship between the light intensity and physical force components $F_{x}, F_{y}$, and $F_{z}$. Using the calibration device, we compute the decoupling stiffness matrix of the linear fitting function for a proper prediction of the applied force components from the calibration data.

\section{1) Calibration Device}

The calibration device consists of a linear guide, a sensor fixture, a sensor base, a load fixture, an actuator, a load cell (six-axis force sensor, ATI Nano 17 IP65). For the calibration, the developed three axis force sensor was mounted on the calibration device as shown in Figs. 10 to 12 . For $F_{z}$ calibration, the load fixture with the six-axis force sensor moves by the actuator to apply physical load on our proposed

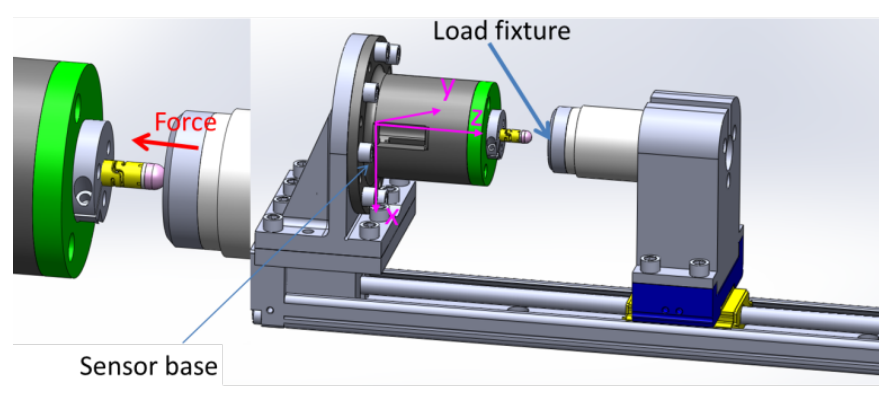

Fig. 10. Overview of calibration device designed by Solidworks to obtain the characteristic curve between the physical force $F_{z}$ and the three light intensity values for the three optical fibers

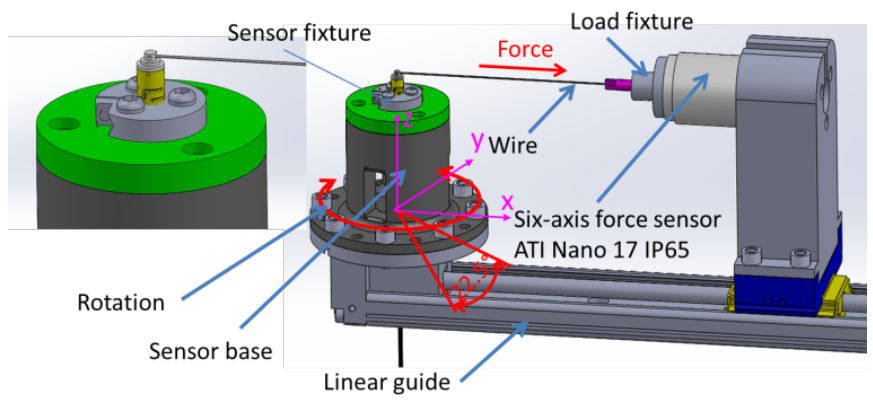

Fig. 11. Overview of calibration device designed by Solidworks to obtain the characteristic curve between the physical force $\left(F_{x}\right.$ and $\left.F_{y}\right)$, and the three light intensity values for the three optical fibers 


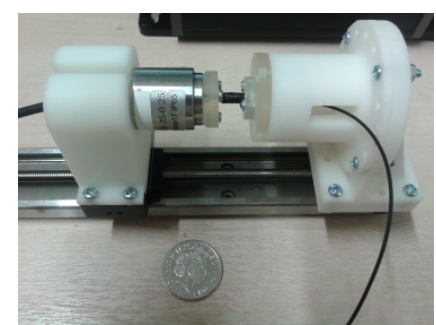

(a)

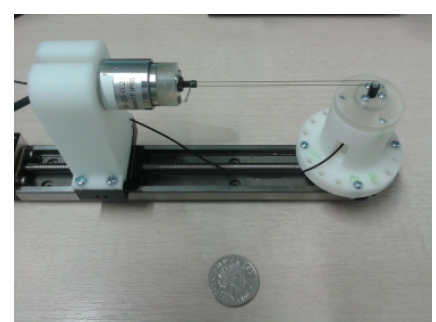

(b)
Fig. 12. Overview of calibration device to obtain the characteristic curve between the physical force and the three light intensity values for the three optical fibers: a) the characteristic curve between $F_{z}$ and the three light intensity value b) the characteristic curves between $\left(F_{x}\right.$ and $\left.F_{y}\right)$ and the three light intensity values

three-axis force sensor as shown in Figs. 10 and 12a. On the other hand, for $F_{x}$ and $F_{y}$ calibration, another type of the sensor base is attached as shown in Fig. 11 and 12b. The sensor base can be rotated in increment of $22.5^{\circ}$, and the physical load can be applied along $\mathrm{x}$-axis direction and $\mathrm{y}$-axis direction by the actuator. For $F_{x}$ and $F_{y}$ calibration, as shown in Fig. 11 and 12b, the load fixture moves backward to apply the physical load on the three axis force sensor by a wire which is connected to the load fixture and the six axis force sensor. This calibration device recorded the six-axis force sensor data and light intensity values refined by Kalman filter in real-time when a physical load is applied by an actuator via the linear guide. Consequently, the relationship between the physical load and the light intensity values can be obtained.

\section{1) Characteristic Curves between Light Intensity Analog Values and Physical Loads}

The calibration result are shown in Figs. 13 to 15 where the physical loads $F_{z}, F_{x}$ and $F_{y}$ were exerted along the linear guide. These characteristic curves express the relationship between the light intensity values and physical load $\left(F_{\mathrm{z}}=0\right.$ to $-1.5 \mathrm{~N}, F_{\mathrm{x}}=-0.5$ to $0.5 \mathrm{~N}$, and $F_{\mathrm{y}}=-0.5$ to $\left.0.5 \mathrm{~N}\right)$ which is exerted by the calibration device. The characteristic curves are fairly linear.

\section{B. Calculation of Force Components by Multiple Linear Regression}

Multiple linear regression aims at finding the relationship between two or more variables and a response variable by means of fitting a linear equation to the observed data [30]. In this implementation, every value of an independent variable namely each value of the three output analog value of the light intensity - is associated with values of the dependent variables - namely the force $F_{z}, F_{x}$, and $F_{y}$. By applying the multiple linear regression, decoupling stiffness matrix can be calculated from each of the three analog samples of the light intensity $\left(a_{1}, a_{2}\right.$, and $\left.a_{3}\right)$ and force components $\left(F_{x}, F_{y}\right.$ and $\left.F_{z}\right)$ as shown in Eqs. (8-9). According to this calculation, the estimated $F_{z}, F_{x}$ and $F_{y}$ agree well with the benchmark force $F_{z}, F_{x}$, and $F_{y}$, as shown in Fig. 16 . It can be noticed that $F_{y}$ has slightly higher crosstalk when compared with the estimation of $F_{z}$ and $F_{x}$, and has higher error on estimation $F_{\mathrm{x}}$ and $F_{y}$. This could be due to the asymmetrical arrangement of the receiving fibers used in our design as shown in Fig. 17a, and it caused characteristic curves to have non-linearity as shown in Figs. 13 to 15. Although the estimation force has a small deviation to the benchmark forces due to the asymmetrical arrangement of the three receiving fibers, the estimated forces in general have a good agreement with the benchmark forces as shown in Fig 16. When the maximum force is applied on the three axis force sensor, the maximum error of force estimation is $F_{x}(+19 \%,-34 \%), F_{y}(+41 \%,-13 \%)$, and $F_{z}(13 \%)$. The performance of our proposed sensor can be ever further improved if we use symmetrical fibers arrangement of the three receiving fibers as shown in fig. $17 \mathrm{~b}$.

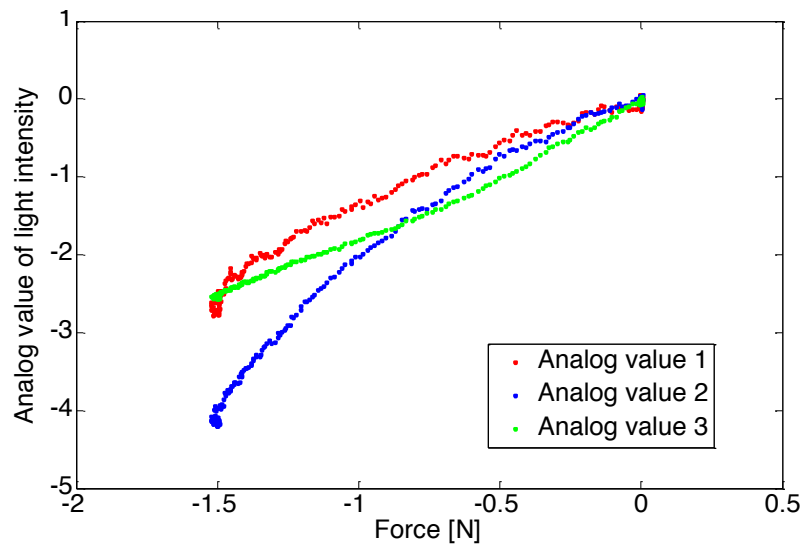

Fig. 13. Characteristic curve between physical force $F_{z}$ and three light intensity values of the three receiving light fibers.

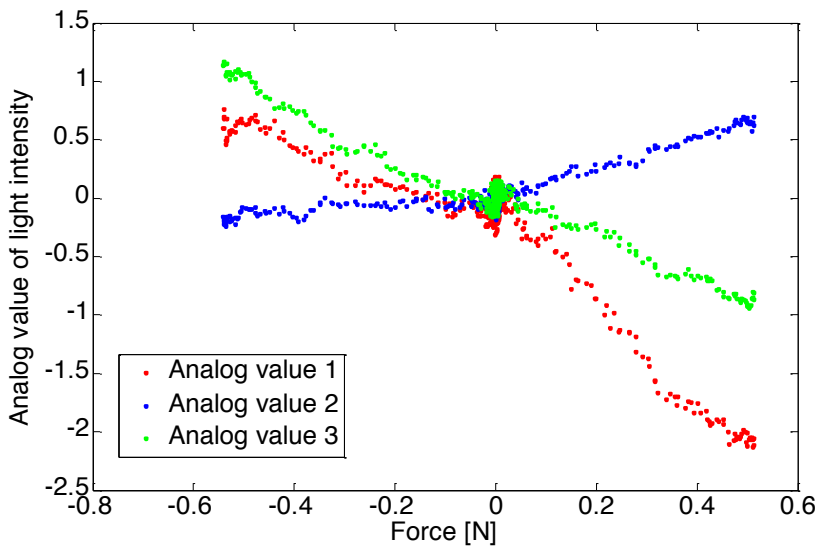

Fig. 14. Characteristic curve between physical force $F_{x}$ and three light intensity values of the three receiving light fibers.

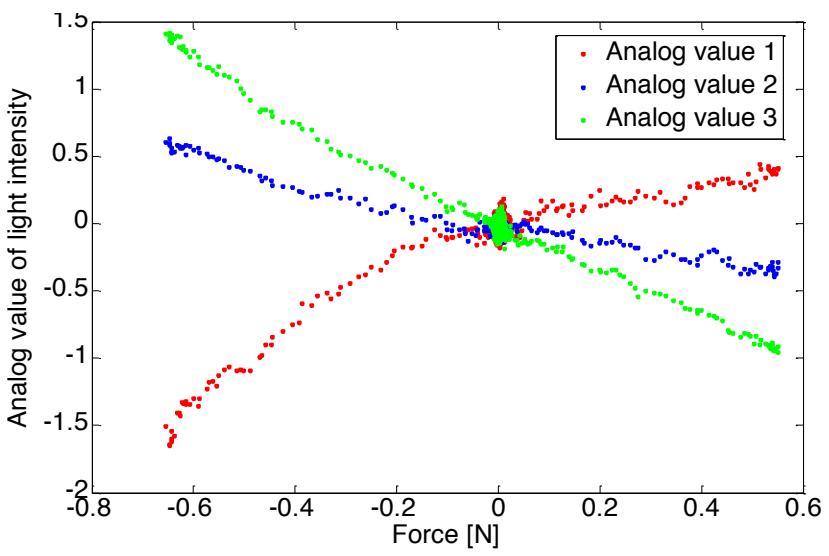

Fig. 15. Characteristic curve between physical force $F_{y}$ and three light intensity values of the three receiving light fibers. 


$$
\begin{gathered}
{\left[\begin{array}{ccc}
k_{1,1} & k_{1,2} & k_{1,3} \\
k_{2,1} & k_{2,2} & k_{2,3} \\
k_{3,1} & k_{3,2} & k_{3,3}
\end{array}\right] \times\left[\begin{array}{l}
a_{1} \\
a_{2} \\
a_{3}
\end{array}\right]=\left[\begin{array}{l}
F_{x} \\
F_{y} \\
F_{z}
\end{array}\right]} \\
K=\left[\begin{array}{ccc}
-0.1094 & 0.2160 & -0.2151 \\
0.1848 & 0.0635 & -0.2706 \\
0.1018 & 0.3154 & 0.0222
\end{array}\right]
\end{gathered}
$$

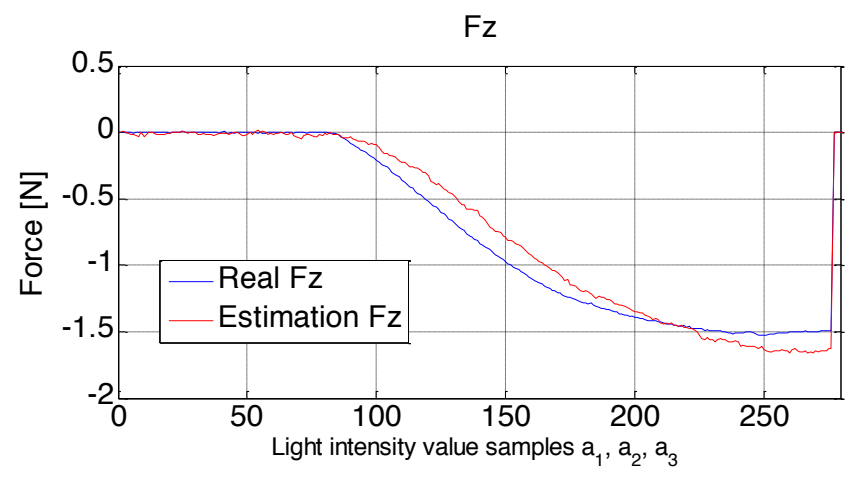

(a)
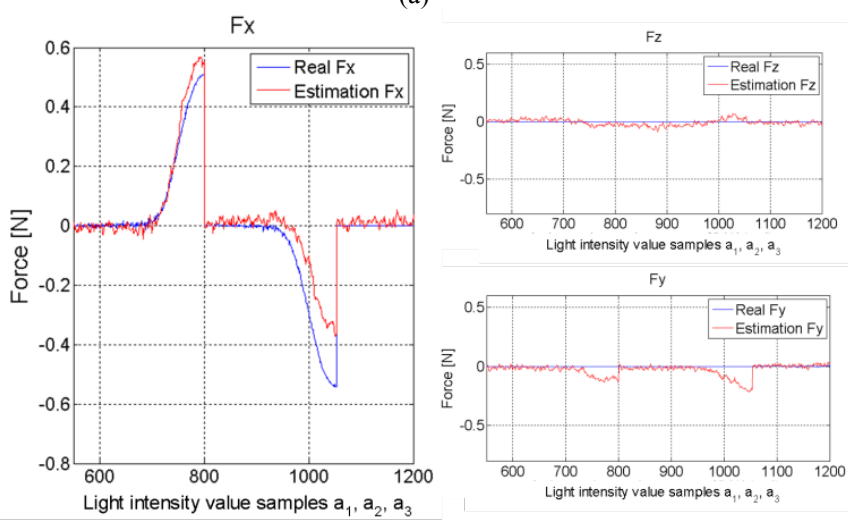

(b)
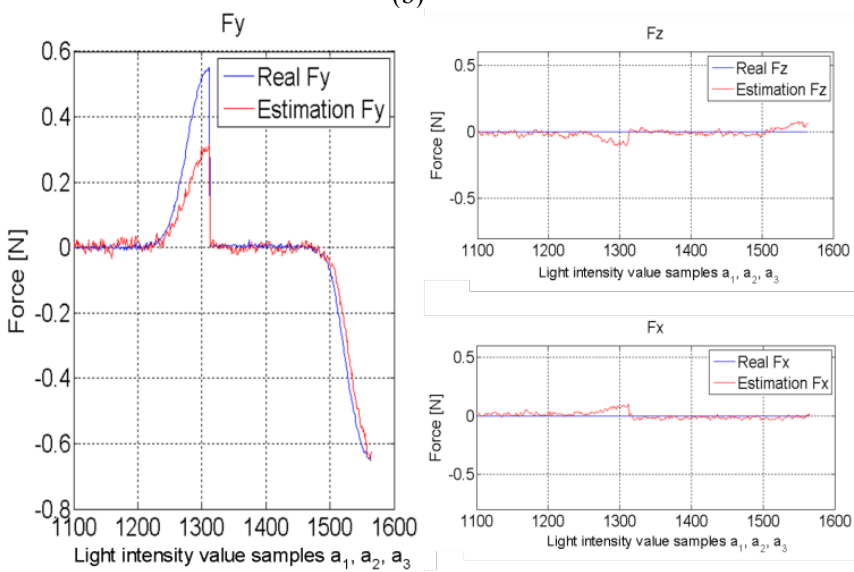

(c)

Fig. 16. Verification of sensor performance test: a) comparison between real $F_{z}$ and estimation $F_{z}$ b) comparison between real $F_{x}$ and estimation $F_{x}$ (cross-talk in other force components $F_{y}$ and $F_{z}$ ) c) comparison between real $F_{y}$ and estimation $F_{y}$ (cross-talk in other force components $F_{x}$ and $F_{z}$ )

\section{CONCLUSION AND FUTURE WORKS}

In this paper, we have presented details of an innovative new three axis force sensor based on an optical fiber, camera vision, and digital image processing (digital filter) to measure external forces applied to the catheter's tip. We have demonstrated how to design a sensor structure which enables multi axis sensing in the catheter's tip, and we have described how to reduce noise, how to carry out calibration, and how to calculate decoupling stiffness matrix by multiple linear regression. Finally, we have also validated the effectiveness of our proposed three axis force sensor through a set of experiments. Our first prototype of the novel sensor approach has achieved $3.5 \mathrm{~mm}$ in diameter with the optical fiber FU49X (diameter ( $\phi 1.45)$, as shown in Fig. 2). The conventional catheters are around 7-7.5 French $(2.31-2.475 \mathrm{~mm})$ in diameter. In extreme case of the ablation, catheters are around 8-10 French (2.64-3.3mm) thick. Hence, in order to integrate our sensor development approach into the catheters (including EP and ablation Catheters) and secure some space to pass electric cables through, it is essential to miniaturize the overall size of the sensor structure. This can be done through reducing the diameter of the optical fiber bundle; the size of a bundle of the multi-cores can be further miniaturized by using a 0.125 $\mathrm{mm}$ optical fiber bundle which is available off the shelf. The diameter of the catheter tip could be further reduced through optimization of the material properties of the sensing structure.

In the future work, we will use MR-compatible materials such as titanium and cobalt-chromium to minimize the catheter's tip size to around 7-7.5 French (2.31-2.475 mm).

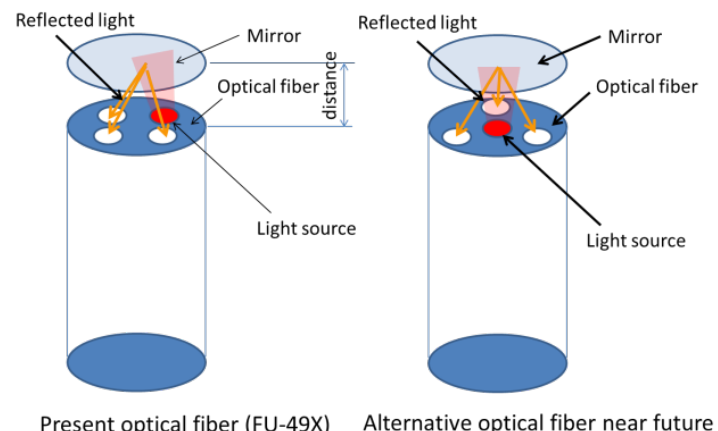

(a)

(b)

Fig. 17. Overview of the three -axis force sensors for catheter: a) present optical fiber which is using for the three axis force sensor for the catheter's tip b) alternative optical fiber for near future

The estimation of the force components calculated by the decoupling stiffness matrix has considerable errors in comparison with the benchmark forces. We think the estimation errors may be caused by the following factors: 1) noise due to external light; 2) distance between the optical fibers and the mirror as shown in Fig. 17; 3) configuration of the optical fiber deployment as shown in Fig. 17;4) camera quality; 5) Kalman filter coefficients; 6) material property.

In 1), the external light makes the light intensity of the camera image unstable, and it produces a small amount of 
noise, and causes the estimation error. Although the black color is painted around the three axis sensor for the catheter tip and the camera box including as shown in Fig. 5, the external light influences the light intensity value. In 2) the light intensity value change and linearity with respect to the deflection of the sensor structure are different depending on the distance between the mirror and the optical fiber, so the distances should be optimized. In 3), the optical fiber (Keyence Corp., FU-49X) is deployed as shown in Fig. 17a. The fibers are not deployed symmetrically with respect to the optical fiber for the light source. It also causes a large amount of the noise and non-linearity (Figures 13 to 15). In 4) for the first prototype, a low resolution $(640 \times 480)$ and low frame rate (30fps) USB camera is used. Using a high-quality CMOS camera or providing a higher image input rate to the image processing approach can potentially reduce the light intensity noise, leading to a reduced force estimation error. We will investigate this further in our future research. However, there will be always a trade-off between the image quality and the image input rate. In 5) the light intensity noise is different depending on each of the three receiving fibers, so each of the Kalman filter coefficients on the three receiving fibers should be optimized. In 6), the sensor structure is made out of a UV curable acrylic plastic (VisiJet ${ }^{\circ E X 200)}$ manufactured by a rapid prototyping machine (Projet 3000, 3D SYSTEM Co., Ltd), with relatively large hysteresis values. The hysteresis influences the sensor's linearity and, hence, negatively impacts the accuracy of calibration matrix, resulting in sensor value estimation errors. In addition, the change of temperature may produce small displacements in the sensing structure and, therefore, affect the sensor readings. The use of MRcompatible materials such as titanium and cobalt-chromium in future designs can potentially reduce the error due to hysteresis and temperature change.

The experimental calibration matrix described in equation (8) and (9) could be potentially ill-conditioned. In order to avoid such drawbacks, in the future, we will carry out an FEM analysis to obtain an initial theoretical calibration matrix under a controlled condition. This process will allow optimisation of the sensor structure for an improved condition number. The calibration matrix will be then fine-tuned using experimental data. This should be mentioned that the sensing error was validated along a single axis at once only. The future work will investigate the sensor error in combination of the three axes.

As a future task, we will investigate possibilities for enhancing the robustness of the sensing system and reducing the estimation errors. Particularly, we will further study the contributions of aforementioned six factors into the total force estimation error and propose appropriate solutions for improved sensing. Moreover, we will further investigate sensor characteristics such as nonlinearity, crosstalk, and repeatability.

\section{REFERENCES}

[1] M. M. Scheinman, F. Morady, D. S. Hess, and R. Gonzalez, "Catheterinduced ablation of the atrioventricular junction to control refractory supra-ventricular arrhythmias,” J. Amer. Med. Assoc., vol. 248, pp. 851-855, 1982.

[2] M. R. Karch, B. Zrenner, I. Deisenhofer, J. R. Schreieck, G. Ndrepepa, J. Dong, K. Lamprecht, P. Barthel, E. Luciani, A. Schomig, and C. Schmitt, "Freedom from atrial tachyarrhythmias after catheter ablation of atrial fibrillation-A randomized comparison between 2 current ablation strategies," Circulation, vol. 111, no. 22, pp. 2875-2880, 2005.

[3] H. Oral, C. Pappone, A. Chugh, E. Good, F. Bogun, F. Pelosi, E. R. Bates, M. H. Lehmann, G. Vicedomini, G. Augello, E. Agricola, S. Sala, V. Santinelli, and F. Morady, "Circumferential pulmonary-vein ablation for chronic atrial fibrillation," N. Engl. J. Med., vol. 354, no. 9, pp. 934-941, 2006.

[4] K. Yokoyama, H. Nakagawa, D. C. Shah, H. Lambert, G. Leo, N. Aeby, A. Ikeda, J. V. Pitha, T. Sharma, R. Lazzara, and W. M. Jackman, "Novel contact force sensor incorporated in irrigated radiofrequency ablation catheter predicts lesion size and incidence of steam pop and thrombus," Circulation, vol. 1, no. 5, pp. 354-362, 2008.

[5] S. R. Dukkipati, R. Mallozzi, E. J. Schmidt, G. Holmvang, A. d'Avila, R. Guhde, R. D. Darrow, G. Slavin, M. Fung, Z. Malchano, G. Kampa, J. D. Dando, C. McPherson, T. K. Foo, J. N. Ruskin, C. L. Dumoulin, and V. Y. Reddy, "Electroanatomic mapping of the left ventricle in a porcine model of chronic myocardial infarction with magnetic resonance-based catheter tracking," Circulation, vol. 118, pp. 853-862, 2008.

[6] P. Polygerinos, L.D. Seneviratne, R. Razavi, T. Schaeffter, K. Althoefer, K.," Triaxial Catheter-Tip Force Sensor for MRI-Guided Cardiac Procedures," IEEE/ASME Transaction on Mechatronics, vol. 18, no. 1, pp. 386-396, 2013.

[7] P. Polygerinos, D. Zbyszewski, T. Schaeffter, R. Razavi, L. D. Seneviratne, and K. Althoefer, "MRI-Compatible Fiber-Optic Force Sensors for Catheterization Procedures," IEEE Sensor Journal, vol. 10, no. 10, pp. 1598-1608, Oct., 2010.

[8] P. Polygerinos, A. Ataollahi, T. Schaeffter, R. Razavi, L. D Seneviratne, K. Althoefer,"MRI-compatible intensity-modulated force sensor for cardiac catheterization procedures, "IEEE Transactions on Biomedical Engineering, vol. 58, Issue 3, pp. 721-726, 2011.

[9] T. Katsumata, Y. Haga, K. Minami, M. Esashi, "Micromachined $125 \mu \mathrm{m}$ diameter ultra miniature fibre-optic pressure sensor for catheter," Transactions IEE Japan, vol. 120, no. 2, pp. 58-63, 2000.

[10] S.W. Hetts, M. Saeed, A.J. Martin, L. Evans, A.F. Bernhardt, V. Malba, F. Settecase, L. Do, E.J. Yee, A. Losey, R. Sincic, P. Lillaney, S. Roy, R.L. Arenson, and M.W. Wilson," Endovascular Catheter for Magnetic Navigation under MR Imaging Guidance: Evaluation of Safety In Vivo at 1.5T," AJNR Am J Neuroradiol vol.34 no.11, pp. 2083-91, 2013.

[11] D. Tanase, J. F. L. Goosen, P. J. Trimp, and P. J. French, "Multiparameter sensor system with intravascular navigation for catheter/guide wire application," Sens. Actuators A, pp. 116-124, 2002.

[12] H. Allen, K. Ramzan, J. Knutti, and S. Withers, "A novel ultraminiature catheter tip pressure sensor fabricated using silicon and glass thinning techniques," in Proc. Materials Research Society. Symposium, San Francisco, pp. 146-151, 2001.

[13] W. Feng, H. Wang, S. Guo, K. Wang, and X. Ye, "Design and experiments of a catheter side wall tactile sensor for minimum invasive surgery," in Proc. Int. Conf. Mechatronics Automation, pp. 1073-1078, 2007.

[14] C. Strandman, L. Smithb, L. Tenerzb, and B. Hök, "A production process of silicon sensor elements for a fiber-optic pressure sensor," Sens. Actuators A, vol. 63, no. 1, pp. 69-74, 1997.

[15] E. Cibula, D. Donlagic, and C. Stropnik, "Miniature fiber optic pressure sensor for medical applications," in Proc. IEEE Sens., pp. 711-714, 2002.

[16] H. Su, G. S. Fischer," A 3-Axis Optical Force/Torque Sensor for Prostate Needle Placement in Magnetic Resonance Imaging Environments," IEEE International Conference on Technologies for Practical Robot Applications (TePRA 2009), pp. 5-9, 2009.

[17] Sareh, S., Noh, Y., Li, M., Ranzani, T., Liu, H., Althoefer, K. (2015) Macro-bend optical sensing for pose measurement in soft robot arms, Smart Mater. Struct. 24125024.

[18] Sareh, S., Noh, Y., Ranzani, T., Wurdemann, H., Liu, H., Althoefer, K., (2015) A 7.5mm Steiner chain fiber-optic system for multi-segment flex sensing, IEEE/RSJ International Conference on Intelligent Robots and Systems (IROS), 2336-2341. 
[19] Noh, Y., Sareh, S., Wudermann, H., Liu, H., Althoefer, K., (2015) Three Axial Fiber-Optic Body Force Sensor for Flexible Manipulators, Sensors-11652-2015.

[20] C. R. Giles, "Lightwave applications of fiber Bragg gratings," J. Lightwave Technology, vol. 15, no. 8, pp. 1391-1404, 1997.

[21] S. Ryu, and P. E. Dupont, "FBG-based Shape Sensing Tubes for Continuum Robots," IEEE International Conference on Robotics \& Automation, pp. 3531-3537, 2014.

[22] Y. Park, US8649847 B1-"Steerable shape sensing biopsy needle and catheter," 2014.

[23] S. Elayaperumal, J. Bae, D. Christensen, M. R. Cutkosky, B. L. Daniel, R. J. Black, J. M. Costa, F. Faridian, B. Moslehi," MR-compatible biopsy needle with enhanced tip force sensing", World Haptics Conference (WHC), pp. 109-114, 2013.

[24] F. Taffoni, D. Formica, P. Saccomandi, G. D. Pino, and E. Schena,"Optical Fiber-Based MR-Compatible Sensors for Medical Applications: An Overview," Sensors 2013, vol.13, no. 10, 1410514120; doi:10.3390/s131014105.

[25] M. Ohka, J. Takata, H. Kobayashi, H. Suzuki, N. Morisawa and H. B. Yussof,"A Robotic Finger Equipped with an Optical Three-axis Tactile Sensor," 2008 IEEE International Conference on Robotics and Automation, pp. 3425 - 3430, 2008.

[26] M. Tada, T. Kanade," Design of an MR-compatible three-axis force sensor," IEEE/RSJ International Conference on Intelligent Robots and Systems, pp. 3505-3510, 2005.

[27] H. Xie, H. Liu, L.D. Seneviratne, K. Althoefer,"An Optical Tactile Array Probe Head for Tissue Palpation During Minimally Invasive Surgery," Sensors Journal, IEEE, vol.14, no.9, pp.3283,3291, Sep. 2014.

[28] H. Xie, A. Jiang, H. A. Wurdemann, H. Liu, L. D. Seneviratne, K. Althoefer, "Magnetic Resonance-Compatible Tactile Force Sensor Using Fiber Optics and Vision Sensor," IEEE Sensors Journal, vol.14, no.3, pp. 829-838, 2014.

[29] R. Faragher," Understanding the Basis of the Kalman Filter Via a Simple and Intuitive Derivation," IEEE Signal Processing Magazine, pp. 128-132, Sep 2012.

[30] L. L. Nathans, F. L. Oswald, K. Nimon, "Interpreting Multiple Linear Regression: A Guidebook of Variable Importance," Practical Assessment, Research \& Evaluation, Vol. 17, No. 9, 2012. 